\title{
Pancreatitis necrohemorrágica en un niño de 11 años. Caso clínico
}

\author{
Necrotizing pancreatitis in a 11 year-old boy. Case report
}

\author{
Dra. Ana Muñoz Lozón ${ }^{a}$, Dra. Cristina Iglesias Blázqueza , Dra. Cristina Menéndez Arias ${ }^{a}$ y \\ Dr. José Manuel Gutiérrez Dueñas ${ }^{b}$
}

\section{RESUMEN}

La pancreatitis aguda es un problema emergente, con una incidencia de 3,6-13,2 casos/100 000 niños. Sin embargo, la pancreatitis necrotizante (necrosis mayor del $30 \%$ del páncreas y/o mayor de $3 \mathrm{~cm}$ de un área del páncreas) es un proceso poco frecuente $(<1 \%$ de las pancreatitis agudas), con presentación similar a los casos de pancreatitis no complicada y con una elevada morbimortalidad. La tomografía axial computada permite valorar, en estos casos, la gravedad del cuadro y el riesgo de complicaciones (puntaje de Balthazar). Las pancreatitis no complicadas tienen una evolución favorable, pero los casos de pancreatitis necrotizante precisan tratamiento médico intensivo y, en ocasiones, cirugía.

Presentamos el caso de un varón de 11 años con clínica compatible con una pancreatitis aguda confirmada mediante ecografía abdominal. Ante el empeoramiento analítico, se realizó una tomografía axial computada, que mostró una pancreatitis necrotizante y pseudoquistes de gran tamaño. Se decidió la monitorización estrecha y la adopción de una actitud conservadora, con mejoría clínico-analítica. Después de un año de seguimiento, el paciente permanece asintomático y han desaparecido los pseudoquistes.

La pancreatitis necrohemorrágica es una entidad infrecuente en la edad pediátrica pero potencialmente graveque debemos tener presente en el diagnóstico diferencial de un abdomen agudo. Palabras clave: pancreatitis aguda, pancreatitis necrotizante, pseudoquistes, niños.

\section{ABSTRACT}

Acute pancreatitis is an emerging problem with an incidence between 3.6 and 13.2 cases $/ 100,000$ children. However, necrotizing pancreatitis (necrosis greater than $30 \%$ of the pancreas and / or greater than $3 \mathrm{~cm}$ in an area of the pancreas) is a rare condition ( $<1 \%$ of acute pancreatitis), with a presentation similar to not complicated pancreatitis cases and with high morbidity and mortality. Computed tomography allows an assessment of the severity of the disease and the risk of complications (Balthazar Score). Not complicated pancreatitis cases have a favorable outcome, but necrotizing pancreatitis cases require intensive medical treatment and sometimes surgical treatment.

a. Servicio de Pediatría del Complejo Asistencial de León.

b. Servicio de Cirugía Infantil del Complejo Asistencial de Burgos.

España.

Correspondencia:

Dra. Ana Muñoz Lozón: amunozlozon@gmail.com

Conflicto de intereses: ninguno que declarar.

Recibido: 2-7-2014

Aceptado: 8-9-2014
We report the case of an 11 year-old boy with clinical presentation compatible with acute pancreatitis and confirmed by abdominal ultrasonography. Due to worsening of laboratory test, an abdominal computed tomography was performed showing a necrotizing pancreatitis and large pseudocysts. Close monitoring and a conservative approach was adopted, with clinical and analytical improvement. After one year followup, the patient is asymptomatic, normal ancillary tests and no evidence of pancreatic pseudocyst.

The pediatric necrohaemorragic pancreatitis is an uncommon and potentially severe entity; we must keep this complication in mind for an adequate differential diagnosis of acute abdomen. Key words: acute pancreatitis, necrotizing pancreatitis, pseudocysts, children.

http:/ /dx.doi.org/10.5546/aap.2015.e39

\section{INTRODUCCIÓN}

La pancreatitis aguda (PA) es la enfermedad pancreática más frecuente en la infancia y se produce por la activación, liberación y autodigestión de la glándula por sus enzimas. La PA es un problema emergente, ${ }^{1,2,3}$ con una incidencia de 3,6-13,2 casos / 100000 niños. ${ }^{4}$ La etiología en niños es variable; las causas más frecuentes son las infecciones víricas, enfermedades sistémicas, traumatológicas, idiopáticas y litiásicas. ${ }^{5}$ Sin embargo, la pancreatitis necrotizante (necrosis mayor del $30 \%$ del páncreas y/o mayor de $3 \mathrm{~cm}$ de un área del páncreas) es un proceso poco frecuente $(<1 \%$ de las PA). ${ }^{1}$

\section{CASO CLÍNICO}

Presentamos el caso de un varón de 11 años que consulta por vómitos de tres días de evolución e intenso dolor abdominal en hipocondrio izquierdo y epigastrio. Como antecedentes familiares, encontramos que la madre había padecido una pseudoobstrucción intestinal; el padre, una pancreatitis aguda litiásica; y el tío paterno, una pancreatitis aguda posiblemente secundaria a consumo de alcohol. Nuestro paciente había sido intervenido de peritonitis secundaria a apendicitis a los 3 años de vida. La exploración física constata un peso de $52,7 \mathrm{~kg}$ (p 90), con un índice de masa corporal de $22,8 \mathrm{~kg} / \mathrm{m}^{2}(\mathrm{p}>97)$, discreta defensa 
a la palpación abdominal y disminución de los ruidos hidroaéreos. En la analítica, se observa leucocitosis con desviación izquierda (leucocitos: $22400 / \mu \mathrm{L}$; neutrófilos: $77 \%$; y cayados: $10 \%$ ) y elevación de amilasa y proteína $C$ reactiva (PCR) (766 U/L y 331,6 mg/L, respectivamente). La radiografía de abdomen en bipedestación muestra niveles hidroaéreos en marco colónico derecho. Se realiza una ecografía abdominal, que detecta una colección líquida en la cabeza del páncreas, otra en el cuerpo y barro biliar. Ante el diagnóstico de PA con sospecha de absceso pancreático, el paciente ingresa a dieta absoluta, con sonda nasogástrica, hidratación endovenosa, analgesia y cobertura antibiótica con amoxicilina-clavulánico. Tras 24 horas, se objetiva aumento de lipasa y PCR (175 U/L y 431,7 mg/L, respectivamente). Se realiza TAC (Figura 1), que muestra una pancreatitis necrohemorrágica (30$50 \%$ de necrosis), por lo que se amplía el espectro antimicrobiano con metronidazol y meropenen. Se canaliza la vena yugular y comienza nutrición parenteral.

Ante la presencia de pseudoquistes (tamaño máximo: $8,2 \times 5,3 \times 7,5$ ), se deriva a un hospital quirúrgico de referencia para valorar drenaje percutáneo o cirugía urgente si precisara. Durante su ingreso, se mantiene estable, y se adopta una actitud conservadora. Presenta disminución de valores analíticos y del tamaño de los pseudoquistes. Se mantiene nutrición

FIGURA 1. Tomografía axial computada: puntaje de Balthazar grado E. Colecciones con un 30-50\% de necrosis

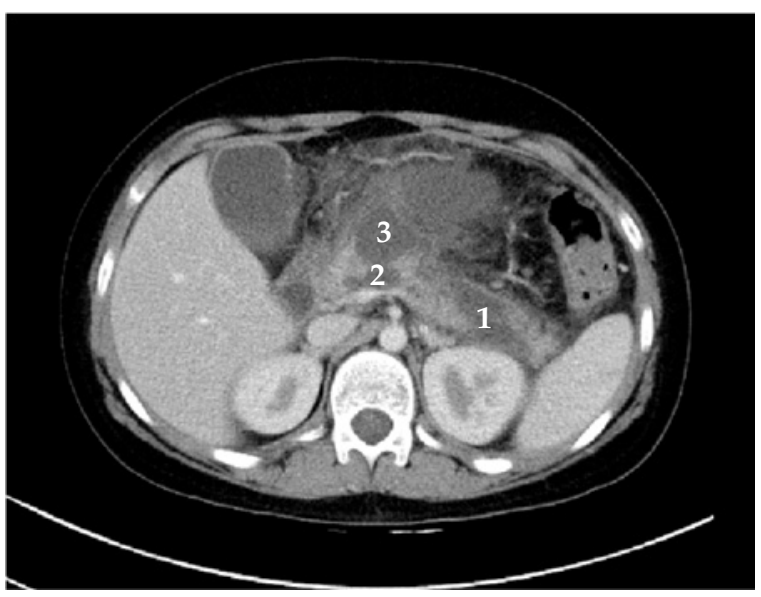

1. Fractura de páncreas a nivel de la unión cuerpo-cola.

2. Colecciones entre las zonas fracturadas.

3. Colección peripancreática. parenteral por 5 días y se reintroduce en forma progresiva la alimentación oral. Se da de alta tras 20 días. Posteriormente, durante su seguimiento en consulta, se constata normalización de las enzimas pancreáticas a los dos meses y medio, y desaparición total de los pseudoquistes a los 3 meses. El paciente se encuentra en seguimiento desde febrero de 2013 hasta la actualidad por el Servicio de Gastroenterología Infantil.

Dentro del estudio etiológico, se ha solicitado serología de virus, bacterias, autoinmunidad, despistaje de enfermedades por depósito, ionotest y colangio RM (Figura 2): todas normales, excepto la presencia de hipertrigliceridemia. La elevación de triglicéridos fue transitoria y se han normalizado las cifras sin necesidad de tratamiento farmacológico. Si volviera a detectarse hipertrigliceridemia, se podría solicitar el estudio genético. Actualmente, el paciente realiza una dieta baja en grasas, permanece asintomático y con perfil lipídico normal. Se están llevando a cabo ecografías seriadas para valorar colecistectomía en el caso de aparición de litiasis biliar.

\section{COMENTARIOS}

La pancreatitis necrotizante tiene una presentación clínica similar a los casos de pancreatitis no complicada, con la clásica triada de dolor abdominal, anorexia y vómitos incoercibles, con o sin fiebre. ${ }^{1}$ El dolor abdominal suele ser de inicio brusco, continuo y ocasiona una posición antiálgica. En casos graves, puede aparecer una coloración azulada periumbilical (signo de Cullen) y en los flancos (signo de Grey Turner) o desarrollarse derrame pleural, ascitis, ictericia e, incluso, un síndrome de respuesta inflamatoria sistémica con fallo multiorgánico y elevada morbimortalidad (en torno a $17 \%){ }^{1,2} \mathrm{La}$ determinación de amilasa y lipasa es la prueba más útil en presencia de una clínica compatible, sin correlacionarse sus niveles con la gravedad del cuadro. ${ }^{1,6}$ La ecografía abdominal es la primera prueba de imagen por realizar, y se reserva la TAC para los casos de evolución desfavorable. La gravedad y el riesgo de complicaciones asociadas puede valorarse mediante TAC según el puntaje de Balthazar, que otorga una puntuación en función del grado de inflamación y de necrosis (de menor grado, A, a mayor, E). ${ }^{1}$ Varios estudios señalan que el puntaje de Balthazar es superior a los sistemas de puntuación clínica clásicamente utilizados, como los de DeBanto ${ }^{2,6,7}$ (Tabla 1). Los casos no complicados suelen evolucionar 
favorablemente en 2-5 días. Sin embargo, los pacientes con pancreatitis necrotizante precisan estancias hospitalarias prolongadas y tratamiento intensivo con dieta absoluta, nutrición parenteral, analgesia y cobertura antibiótica. ${ }^{1}$ El uso de antibioterapia profiláctica es controvertido, aunque se recomienda cuando la necrosis es superior al $30 \%$ por incremento del riesgo de infección. ${ }^{8}$ La presencia de pseudoquistes aumenta el riesgo potencial de mortalidad. Su drenaje

FIGURA 2. Colangio RM (tras 6 semanas). Atrofia parcial del tejido glandular en el cuerpo (izquierda) y una colección bien definida de 3,7 × 2,1 cm encima de la cola del páncreas, entre el cuerpo gástrico y el bazo (derecha)
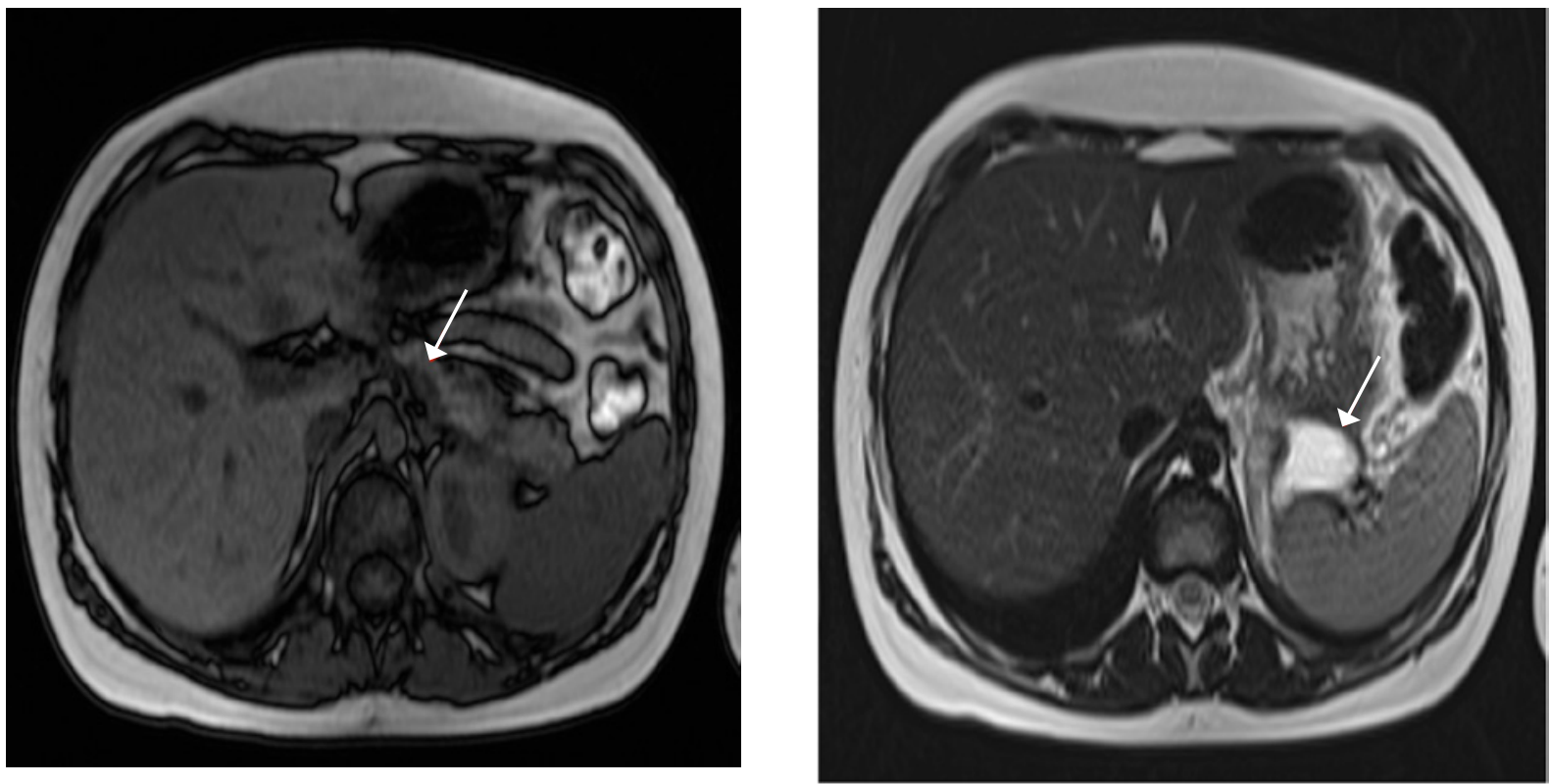

TABLA 1. Escalas utilizadas en la valoración de la gravedad de una pancreatitis

\begin{tabular}{|c|c|c|}
\hline \multicolumn{2}{|c|}{ Puntaje de Balthazar } & \multirow{2}{*}{$\begin{array}{l}\text { Criterios de DeBanto } \\
\text { Edad menor de } 7 \text { años }\end{array}$} \\
\hline Necrosis & Inflamación & \\
\hline O: ninguna & 0: páncreas normal & Peso menor de $23 \mathrm{~kg}$ \\
\hline \multirow[t]{2}{*}{$2:<30 \%$} & 1: pancreatitis edematosa & Secuestro de líquido mayor de \\
\hline & & $75 \mathrm{ml} / \mathrm{kg}$ en las primeras 48 horas \\
\hline 4: $30-50 \%$ & $\begin{array}{l}\text { 2: mayor edema y cambios } \\
\text { extrapancreáticos leves }\end{array}$ & $\begin{array}{c}\text { Más de } 18500 \text { leucocitos } \\
\text { al ingreso }\end{array}$ \\
\hline \multirow[t]{5}{*}{$6:>50 \%$} & 3: cambios extrapancreáticos graves & $\begin{array}{l}\text { Más de } 2000 \text { U/L de láctico } \\
\text { deshidrogenasa al ingreso }\end{array}$ \\
\hline & 4: cambios extrapancreáticos & En las primeras 48 horas: \\
\hline & múltiples o extensas colecciones & - Descenso de calcio a menos de 8,3 mg/dl \\
\hline & & - Descenso de albúmina a menos de 2,6 mg/dl \\
\hline & & - Elevación de nitrógeno ureico en sangre mayor de $5 \mathrm{mg} / \mathrm{dl}$ \\
\hline
\end{tabular}

Puntaje de Balthazar: El índice de gravedad global es la suma del grado de inflamación y del grado de necrosis (rango de 0 a 10). ${ }^{1}$

Criterios de DeBanto: La presencia de tres o más de estos criterios establece la gravedad del cuadro con una sensibilidad del $70 \%$ y una especificidad del $79 \%{ }^{3}$ 
(por vía endoscópica o percutánea) se realiza en casos muy seleccionados y se recomienda en los mayores de $5 \mathrm{~cm}$ de diámetro o cuando persisten más de 3 meses. Los pseudoquistes de menor tamaño, por lo general, se resuelven espontáneamente. El desbridamiento quirúrgico de la necrosis se realiza de forma excepcional. ${ }^{1}$ En el caso de causa litiásica sospechada o probada, se recomienda la extracción de cálculos mediante colangiopancreatografía retrógrada endoscópica o, incluso, la colecistectomía.

Nuestro paciente evolucionó hacia la resolución espontánea sin precisar ningún tipo de intervención. La pancreatitis necrohemorrágica es una entidad infrecuente en la infancia y la adolescencia; sin embargo, debemos tenerla presente en el diagnóstico diferencial de un abdomen agudo. Aunque las pancreatitis agudas complicadas tienen una elevada morbimortalidad, el pronóstico es mejor que en adultos. ${ }^{1}$

\section{REFERENCIAS}

1. Raizner A, Phatak UP, Baker K, Patel MG, et al. Acute necrotizing pancreatitis in children. J Pediatr 2013;162(4):78892.
2. Chang YJ, Chao HC, Kong MS, Hsia SH, et al. Acute pancreatitis in children. Acta Paediatr 2011;100(5):740-4.

3. Solar Boga A. Pancreatitis aguda. En: Argüelles Martín F, García Novo MD, Pavón Belinchón P, Román Riechmann E, et al., eds. Tratado de gastroenterología, hepatología y nutrición pediátrica aplicada de la SEGHNP. Madrid: Ergon; 2011. Págs.386-93.

4. Morinville VD, Husain SZ, Bai H, Barth B, et al. Definitions of pediatric pancreatitis and survey of present clinical practices. J Pediatr Gastroenterol Nutr 2012;55(3):261-5.

5. García Burriel JI, Vilar Escrigas PJ. Pancreatitis en el niño. En Asociación Española de Pediatría, Sociedad Española de Gastroenterología Hepatología y Nutrición Pediátrica, eds. Protocolos diagnóstico-terapéuticos de Gastroenterología, Hepatología y Nutrición Pediátrica. 2. ${ }^{a}$ ed. Madrid: Ergón; 2010.Págs.135-42. [Disponible en: http:/ / www.aeped.es/ sites/default/files/documentos/pancreatitis.pdf]. [Acceso: 5 de mayo de 2014].

6. Fabre A, Petit P, Gaudart J, Mas E, et al. Severity scores in children with acute pancreatitis. J Pediatr Gastroenterol Nutr 2012;55(3):266-7.

7. Lautz TB, Turkel G, Radhakrishnan J, Wyers M, et al. Utility of the computed tomography severity index (Balthazar score) in children with acute pancreatitis. J Pediatr Surg 2012;47(6):1185-91.

8. Working Party of the British Society of Gastroenterology; Association of Surgeons of Great Britain and Ireland; Pancreatic Society of Great Britain and Ireland; Association of Upper GI Surgeons of Great Britain and Ireland. UK guidelines for the management of acute pancreatitis. Gut 2005;54 (Suppl 3):iii1-9. 